\title{
THE SPECTRUM OF LINEAR TRANSFORMATIONS
}

\author{
BY
}

\author{
EDGAR R. LORCH
}

\section{INTRODUCTION}

The determination of the structure of a linear transformation $T$ which maps a complex vector space $\mathfrak{B}$ into itself is naturally a primary object of the theory of linear transformations. The first stage of such a study centers about the question of reducibility. The concept may be introduced in various ways. In one formulation it is required to find all projections (bounded idempotent transformations) $P$ which commute with $T$. At a more incisive level, one seeks those pairs of manifolds $\mathfrak{M}$ and $\mathfrak{R}$ (not necessarily disjoint) which "split" the space and are transformed in to themselves by $T$. For transformations in general vector spaces the known results all refer to special types such as the completely continuous or the weakly almost periodic. This paper will deal almost exclusively with the first type of reducibility of the general bounded transformation. The boundedness of $T$ is assumed for convenience; in case merely its closure is hypothesized the salient features of the theory are still valid.

The results are all based on one method, that of a contour integral of the resolvent of $T$. They seem to exhaust the possibilities for this particular tool. Means for cracking the spectrum directly would undoubtedly have to be of a much more delicate nature. The fundamental projection is the integral

$$
P=\frac{1}{2 \pi i} \int_{C} \frac{d \zeta}{\zeta I-T}
$$

evaluated over a simple closed contour lying entirely in the resolvent set of $T\left(^{1}\right)$. An integral bearing suggestive resemblance to this one is used by E. Hille in an analysis of semi-groups of linear transformations $\left({ }^{2}\right)$. The range of $P$ consists entirely of those elements in $\mathbb{B}$ associated with the spectral val-

Presented to the Society, May 2, 1941; received by the editors March 24, 1941, and, in revised form, July $16,1941$.

(1) This transformation is well known in the theory of finite matrices. It was introduced by Frobenius, Über die schief Invariante einer bilinearen oder quadratischen Form, Crelles Journal, vol. 86 (1879), pp. 44-71. The construction of $P$ and the demonstration of its properties in this case are quite simple and are carried out by methods characteristic of that theory.

(2) E. Hille, Notes on linear transformations. II. Analyticity of semi-groups, Annals of Mathematics, (2), vol. 40 (1939), pp. 1-47. As a principal problem of this paper is one in interpolation, Professor Hille uses an integral of the type $T^{\alpha}=(2 \pi i)^{-1} \int_{C} \zeta^{\alpha}(\zeta I-T)^{-1} d \zeta$ where $\alpha$ is a complex power and $C$ represents a simple closed curve essentially containing the entire spectrum of $T$ in its interior. Thus the emphasis here is on the factor $\zeta^{\alpha}$. In our case, on the contrary, the choice of the curve $C$ is paramount. Added November 12, 1941. 
ues lying within the curve $C$. Similarly the spectrum of $T$ over the range of $I-P$ lies outside of $C$. If one associates the projection $P$ with the point set interior to $C$ one has an instance of a mapping of so-called spectral sets upon projections. It is shown that this mapping is a homomorphism. A spectral set is any set obtained from the interiors of curves $C$ by the operations of complementation, addition, and intersection performed a finite number of times. The attempt to extend the homomorphism so as to admit of denumerable

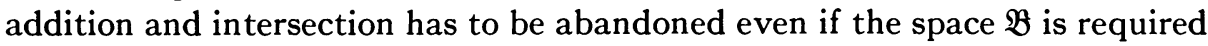
to be reflexive and the more general type of reducibility (vide supra) is considered.

\section{The fundamental projections}

Notations and definitions. The notations used are these: Complex numbers are denoted by $\alpha, \beta, \gamma, \zeta, \lambda, \xi$, and so on ; elements of the space $\mathfrak{B}$ by $f, g, h$, and so on; linear transformations of $\mathfrak{B}$ into a subset of itself by $T, S, I$ (the identity), 0 (the zero), $P$, and so on.

The underlying space $\mathfrak{B}$ is a normed linear complex vector space, that is, a complex Banach space. The transformations $T$ are assumed to be linear or distributive, $T(\alpha f+\beta g)=\alpha T f+\beta T g$. Unless explicitly stated, $T$ is bounded, $\|T f\| \leqq K\|f\|$, for arbitrary $f \in \mathfrak{B}$; the bound of $T$ is denoted by $|T|$.

A number $\lambda$ is said to belong to the resolvent set $R$ of $T$ if the transformation $T-\lambda I$ maps $\mathfrak{B}$ upon itself in a one-to-one manner. It results from this fact that $(T-\lambda I)^{-1}$ is a bounded linear transformation $\left(^{3}\right)$.

If for a given $\lambda, T f-\lambda f=0$ for an $f \neq 0, \lambda$ is said to belong to the point spectrum of $T$. If $\lambda$ belongs neither to the point spectrum nor to the resolvent set, the transformation $T-\lambda I$ may be inverted and has a range which is either dense in $\mathscr{B}$ (but not identical with $\mathfrak{B}$ ), or is not dense in $\mathscr{B}$. In the first case $\lambda$ is said to belong to the continuous spectrum of $T$; in the second, $\lambda$ is said to belong to the residual spectrum of $T$. If $\lambda$ is in the continuous spectrum, the transformation $(T-\lambda I)^{-1}$ is unbounded and there exist elements $f_{n} \in \mathfrak{B}$, $n=1,2, \cdots$, such that $\left\|f_{n}\right\|=1,\left\|(T-\lambda I) f_{n}\right\| \rightarrow 0$. The collection of values $\lambda$ in the point, continuous, and residual spectra is called the spectrum $S$ of $T$. These three classes in addition to the resolvent set are mutually exclusive and all inclusive in the complex plane.

Some elementary theorems. In this section some elementary theorems are stated.

THEOREM 1. The value $\lambda$ is in the resolvent set (spectrum) of $T$ if and only if the value $\bar{\lambda}$ is in the resolvent set (spectrum) of the adjoint $\bar{T}$ of $T$. If $\lambda$ is in the point spectrum of $T, \bar{\lambda}$ is in the point or residual spectrum of $\bar{T}$. If $\lambda$ is in the residual spectrum of $T, \bar{\lambda}$ is in the point spectrum of $\bar{T}$. If $\lambda$ is in the continuous spectrum of $T, \bar{\lambda}$ is in the continuous or residual spectrum of $\bar{T}$. If the space $\mathfrak{B}$

(3) Banach, Thêorie des Opérations Linéaires, p. 41. 
is reflexive and $\lambda$ is in the continuous spectrum of $T$, then, $\bar{\lambda}$ is in the continuous spectrum of $\bar{T}\left({ }^{4}\right)$.

The theorem is an immediate consequence of the definition of the adjoint transformation and of some elemental properties of linear transformations.

THEOREM 2. If $\lambda$ belongs to the resolvent set $R$ of $T$, then so do all $\zeta$ with $|\zeta-\lambda|<1 /\left|(T-\lambda I)^{-1}\right|$. Thus $R$ is an open set and the spectrum $S$ is a closed set. The transformation $(T-\zeta I)^{-1}$ may be expressed "analytically" in terms of $(T-\lambda I)^{-1}$ by means of the formula $\left(^{5}\right)$ :

(1) $(T-\zeta I)^{-1}=(T-\lambda I)^{-1}+(\zeta-\lambda)(T-\lambda I)^{-2}+(\zeta-\lambda)^{2}(T-\lambda I)^{-3}+\cdots$.

The bound of $(T-\zeta I)^{-1}$ is a continuous function of $\zeta$.

No proof will be given.

That the resolvent set is not empty may be shown as follows: Let $\lambda$ be so chosen that $|\lambda|>|T|$. Then since $\|(T-\lambda I) f\|$ is bounded away from zero, $\lambda$ is neither in the point nor in the continuous spectrum. By Theorem 1 and the fact that $|T|=|T|, \lambda$ is not in the residual spectrum. Thus $\lambda \in R$. The fact that $S$ is not empty will be shown later.

THEOREM 3. If $\lambda$ and $\mu$ are any two values in the resolvent set $R$ of $T$, then $\left(^{6}\right)$

$$
(\lambda I-T)^{-1}-(\mu I-T)^{-1}=(\mu-\lambda)(\lambda I-T)^{-1}(\mu I-T)^{-1} .
$$

In proof, multiply both sides of (2) by $(\lambda I-T)(\mu I-T)$.

Let $T(\zeta)$ be a function defined over some set of complex numbers $\zeta$ and whose values are bounded linear transformations of $\mathfrak{B}$ in to itself. For such a transformation depending continuously upon a parameter will be defined a curvilinear integral along a given rectifiable curve $C$. The function $T(\zeta)$ is said to be continuous if it is continuous in the uniform topology, that is, $\left|T\left(\zeta_{1}\right)-T\left(\zeta_{2}\right)\right|$ is small with $\left|\zeta_{1}-\zeta_{2}\right|$.

LEMma. Let $C$ be a rectifiable curve in the complex plane, $\zeta=\phi(t), 0 \leqq t \leqq 1$, and $T(\lambda)$ be a transformation depending continuously upon a parameter $\lambda$ and defined on $C$. Then the Riemann integral

$$
A=\int_{C} T(\zeta) d \zeta
$$

(4) Some of these statements may be found established for Hilbert space by Stone, Linear Transformations in Hilbert Space and Their Applications to Analysis, American Mathematical Society Colloquium Publications, vol. 15, chap. 4.

(5) This is the well known Neumann expansion of the resolvent. The fact that this formula holds for a general transformation in a Banach space has been noted by A. E. Taylor, The resolvent of a closed transformation, Bulletin of the American Mathematical Society, vol. 44 (1938), pp. 70-74. In the same paper one will find our Theorem 3.

(8) This is the well known functional equation for the resolvent. 
exists and defines a bounded linear transformation. The bound of $A$ satisfies $|A| \leqq \max _{C}|T(\zeta)| \cdot l$ where $l$ is the length of $C$. If the transformations $T(\zeta)$ are commutative, then $A$ is commutative with $T(\zeta)$.

The integral is defined in the classic way. Let $t_{0}=0<t_{1}<\cdots<t_{n}=1$ be a subdivision of the unit interval. Let $t_{i}^{\prime}$ with $t_{i-1} \leqq t_{i}^{\prime} \leqq t_{i}, i=1, \cdots, n$ be $n$ points, one in each subinterval. Let $\zeta_{i}^{\prime}=\phi\left(t_{i}^{\prime}\right)$ and $\Delta \zeta_{i}=\phi\left(t_{i}\right)-\phi\left(t_{i-1}\right)$. Then an approximating sum to $A$ is $\sum_{i=1}^{n} T\left(\zeta_{i}^{\prime}\right) \Delta \zeta_{i}$. The proof of the convergence is omitted. The remaining statements of the theorem are clear.

The fundamental projections. In this section will be introduced the projections which underlie the entire investigation.

THEOREM 4. Let $C$ be a simple closed rectifiable curve lying entirely within the resolvent set $R$ of $T$. Then the contour integral

$$
P=\frac{1}{2 \pi i} \int_{C} \frac{d \zeta}{\zeta I-T}
$$

exists and represents a bounded linear transformation commutative with $T$, $P T=T P$. The transformation $P$ is unchanged if the curve $C$ is continuously deformed into a curve $C^{\prime}$, providing only that the deformation is effected without going outside of the resolvent set.

It is hardly necessary to state that the transformation $\zeta I-T$ appearing in the denominator of (4) represents $(\zeta I-T)^{-1}$. By Theorem $2,\left|(\zeta I-T)^{-1}\right|$ is a continuous function of $\zeta$; hence by the lemma, the integral (4) exists defining a bounded transformation $P$. Note that the direction of integration along $C$ is counterclockwise.

Let $\lambda$ be in $R$ and $K$ be a rectifiable closed curve simple or not, lying entirely within the $\zeta$-circle $|\zeta-\lambda|=r<1 /\left|(T-\lambda I)^{-1}\right|$. The integral

$$
\int_{K} \frac{d \zeta}{\zeta I-T}
$$

is computed with the help of series (1); termwise integration shows that its value is zero. Now the curve $C$ may be deformed into the curve $C^{\prime}$ with the help of a finite number of curves of the type $K$. Thus the integral (4) evaluated over $C$ is identical with that integral over $C^{\prime}$.

THeOREM 5. Let $C$ and $C^{\prime}$ be two simple closed rectifiable curves lying entirely within the resolvent set $R$ of $T$. Let $P$ and $P^{\prime}$ be the integrals (4) associated with $C$ and $C^{\prime}$, respectively. If the curve $C$ lies entirely within the curve $C^{\prime}$ then

$$
P P^{\prime}=P^{\prime} P=P \text {. }
$$

If the curves $C$ and $C^{\prime}$ lie each exterior to the other, then

$$
P P^{\prime}=P^{\prime} P=0 \text {. }
$$


That $P P^{\prime}=P^{\prime} P$ is clear from the integral definition of both $P$ and $P^{\prime}$ which brands them as "functions of $T$."

By virtue of Theorem 3, one may write for an arbitrary pair of curves $C$ and $C^{\prime}$

$$
\begin{aligned}
P P^{\prime} & =\left(\frac{1}{2 \pi i}\right)^{2} \int_{C} \int_{C^{\prime}} \frac{1}{\zeta I-T} \cdot \frac{1}{\xi I-T} d \zeta d \xi \\
& =\left(\frac{1}{2 \pi i}\right)^{2} \int_{C} \int_{C^{\prime}}\left(\frac{1}{\zeta I-T}-\frac{1}{\xi I-T}\right) \frac{1}{\xi-\zeta} d \zeta d \xi .
\end{aligned}
$$

If the last integral is broken into two parts, the results of the theorem are readily obtained from a trivial integration in the complex plane.

It should be noted that the conditions imposed by the present theorem on $C$ and $C^{\prime}$ could be weakened without impairing the results (6) and (7). It is sufficient for (6) to require that $C$ and $C^{\prime}$ be deformable within $R$ to two new curves which lie one inside the other. Likewise, (7) holds if $C$ and $C^{\prime}$ are deformable within $R$ to two new curves which lie exterior to each other. All this is possible by virtue of Theorem 4 .

The next theorem announces the most important property of the transformation $P$.

THeOREM 6. The transformation $P$ of Theorem 4 is a projection, that is, $P^{2}=P$. Furthermore, it reduces $T$, that is, $P T=T P$.

Let $C^{\prime}$ be a curve in $R$ such that the curve $C$ defining $P$ lies entirely in the interior of $C^{\prime}$ and such that $C$ and $C^{\prime}$ may be deformed into each other within R. Let $P^{\prime}$ be the transformation (4) associated with $C^{\prime}$. By Theorem 4, $P=P^{\prime}$. By Theorem 5, $P P^{\prime}=P$. Hence $P^{2}=P$.

That $P T=T P$ has already been stated in Theorem 4 . The significance of the role of $P$ relative to $T$ now becomes clear. The space $\mathfrak{B}$ may be thought of as the direct sum of two spaces $\mathfrak{M}$ and $\mathfrak{N}, \mathfrak{B}=\mathfrak{M} \dot{+} \mathfrak{N}$, with $\mathfrak{M}$ the totality of elements $f \in \mathfrak{B}$ for which $P f=f$, and with $\mathfrak{N}$ the totality of elements $g \in \mathfrak{B}$ for which $P g=0$. Since $P T f=T P f=T f, T \mathfrak{M} \subset \mathfrak{M}$. In the same manner $T \mathfrak{N} \subset \mathfrak{R}$. Thus the study of $T$ in $\mathfrak{B}$ reduces to the study of $T$ in the two spaces $\mathfrak{M}$ and $\mathfrak{N}$.

A special case. A special case of considerable interest throws light on the character of the elements $f$ for which $P f=f$ (or $P f=0$ ). This character is completely revealed by the behavior of the sequence of iterates $T^{n} f$.

THEOREM 7. Let the unit circle $C$ with center at the origin of the complex plane lie entirely in the resolvent set $R$ of $T$. Then the projection $P$ defined by (4) satisfies the relation

$$
P=\lim _{n \rightarrow \infty}\left(I-T^{n}\right)^{-1}
$$


Furthermore, $P f=f$ if and only if $\lim _{n \rightarrow \infty}\left\|T^{n} f\right\|=0$. And if $P f=0, f \neq 0$, then $\lim _{n \rightarrow \infty}\left\|T^{n} f\right\|=\infty$.

If $T^{-1}$ exists, the projection associated by (4) to the transformation $T^{-1}$ and for the unit circle $C$ is $I-P$ where $P$ is as above. The elements $f$ for which $P f=0$ are precisely those for which $\lim _{n \rightarrow \infty}\left\|T^{-n} f\right\|=0$.

In addition, if $f(\neq 0)$ is such that $\lim _{n \rightarrow \infty}\left\|T^{n} f\right\|=0$, then $\lim _{n \rightarrow \infty}\left\|T^{-n} f\right\|=\infty$. If $(\neq 0)$ is such that $\lim _{n \rightarrow \infty}\left\|T^{-n} f\right\|=0$ then $\lim _{n \rightarrow \infty}\left\|T^{n} f\right\|=\infty$.

The integral (4) in the special case indicated by the present theorem may be evaluated in the following manner: Let $\alpha=\exp (2 \pi i / n)$. Consider as an approximation to the required integral the finite sum

$$
\frac{1}{2 \pi i} \sum_{j=0}^{n-1}\left(\alpha^{i} I-T\right)^{-1}\left(\alpha^{j+1}-\alpha^{j}\right)
$$

Replace this sum by its equivalents

$$
\frac{1}{2 \pi i} \sum_{j=0}^{n-1}\left(I-\alpha^{-j} T\right)^{-1}(\alpha-1)=\frac{n}{2 \pi i}(\alpha-1)\left(I-T^{n}\right)^{-1} .
$$

Since $\lim _{n \rightarrow \infty} n(\alpha-1)=2 \pi i$, equation (8) has been derived.

The relation

$$
\left(I-T^{n}\right)^{-1}=I+\left(I-T^{n}\right)^{-1} T^{n}
$$

may be verified immediately by multiplication with $\left(I-T^{n}\right)$. It implies that if $\lim _{n \rightarrow \infty}\left\|T^{n} f\right\|=0$, then since $\left|\left(I-T^{n}\right)^{-1}\right|$ is bounded (by (8)), it follows that $\lim _{n \rightarrow \infty}\left(I-T^{n}\right)^{-1} T^{n} f=0$. Applying (9) once more, this means $\lim _{n \rightarrow \infty}\left(I-T^{n}\right)^{-1} f$ $=f$. In resumé, if $\lim _{n \rightarrow \infty}\left\|T^{n} f\right\|=0$, then $P f=f$.

Now, assume that $P f=f$. Write $\left(I-T^{n}\right)^{-1}=Q_{n}$ and let $n_{0}$ be so chosen that $\left|P-Q_{n}\right|<\epsilon<1 / 2$ for $n>n_{0}$. Let $Q_{n} f=g_{n}$. Then since $P T^{n}=T^{n} P, P T^{n} f=T^{n} f$, and (9) may be written

$$
g_{n}-f=T^{n} f+\left(Q_{n}-P\right) T^{n} f .
$$

Upon taking norms, this yields $\epsilon\|f\| \geqq\left\|T^{n} f\right\|-\epsilon\left\|T^{n} f\right\|$. Finally, $\left\|T^{n} f\right\|$ $\leqq \epsilon /(1-\epsilon)\|f\|$ for $n>n_{0}$. This shows that $\lim _{n \rightarrow \infty}\left\|T^{n} f\right\|=0$.

If $P f=0$, (9) yields $Q_{n} f=f+Q_{n} T^{n} f=f+\left(P-Q_{n}\right) T^{n} f+P T^{n} f$, and hence since $P T^{n} f=T^{n} P f=0,\left\|Q_{n} f\right\|+\epsilon\left\|T^{n} f\right\| \geqq\|f\|$. This gives $\left\|T^{n} f\right\| \geqq(1-\epsilon) / \epsilon\|f\|$ which means either $f=0$ or $\lim _{n \rightarrow \infty}\left\|T^{n} f\right\|=\infty$.

If $T^{-1}$ exists, the complex unit circle is in the resolvent set of $T^{-1}$ and (9) shows that $\lim _{n \rightarrow \infty}\left(I-T^{-n}\right)^{-1}=I-P$. Proof of the remaining statements of the theorem may now be based on what has preceded.

Some elementary properties of the fundamental projections. The relationship between the spectrum of $T$ and the projection $P$ is given in the next theorem. 
Theorem 8. The projection $P$ defined by the curve $C$ as in Theorem 4 has the following properties:

(a) If $\lambda$ is in the point spectrum of $T, T f=\lambda f$ with $f \neq 0$, then $P f=f$ or $P f=0$ according as $\lambda$ lies within $C$ or without $C$.

(b) If $\lambda$ is in the continous spectrum and $\left\{f_{n}\right\}$ is a sequence of elements in $\mathfrak{B}$ with the properties $\left\|f_{n}\right\|=1, n=1,2, \cdots, \lim _{n \rightarrow \infty}\left\|T f_{n}-\lambda f_{n}\right\|=0$, then $\lim _{n \rightarrow \infty}\left\|P f_{n}-f_{n}\right\|=0$ if $\lambda$ lies within $C ; \lim _{n \rightarrow \infty}\left\|P_{n} f\right\|=0$ if $\lambda$ lies without $C$.

(c) The equation $P=0$ is valid if and only if every point in the interior of $C$ lies in the resolvent set of $T$.

(d) The equation $P=I$ is valid if and only if every point in the exterior of $C$ is in the resolvent set of $T$.

The proof of (a) is immediate; that of (b) rests on the existence of elements $f$ for which $T f=\lambda f$ approximately. In (c) if the interior of $C$ is in $R$, $C$ may be shrunk to a point, hence $P=0$ by Theorem 4 . If $P=0$, application of (a) and (b) excludes the point and continuous spectrum from the interior of $C$. The residual spectrum is treated by reverting to the space ( $\mathfrak{B})$. For (d), if $P=I$, clearly, the exterior of $C$ lies in $R$. In the converse case, assume $C$ to be a circle with center in $R$ and map its exterior upon the interior of the unit circle, then apply Theorem 7 .

A by-product of this theorem is that every transformation has at least one point in its spectrum, a fact that has already been noted $\left({ }^{7}\right)$.

\section{The HOMOMORPHISM OF SPECTRAL SETS AND PROJECTIONS}

Spectral sets. Let $C$ be a simple closed rectifiable curve lying entirely in the resolvent set of $T$. The curve $C$ determines two sets of points, its interior $\boldsymbol{C}^{i}$, and its exterior $\boldsymbol{C}^{e}$. The totality of sets formed from the sets $\boldsymbol{C}^{i}$ and $\boldsymbol{C}^{e}$ (for all possible curves $C$ ) by the operations of complementation, finite set addition, and finite set intersection forms a Boolean algebra of sets. Any set in this algebra of sets will be called a spectral set of $T$.

With each spectral set $M$ will be associated a projection $P_{M}$ which reduces $T$. The method of assigning $P_{M}$ to $M$ will be the following: To the interior $C^{i}$ of $C$ will be assigned the projection $P$ of Theorem 4. To the exterior $C^{e}$ of $C$ will be assigned the projection $I-P$. To the set complements of $C^{i}$ and $C^{e}$ will be assigned the projections $I-P$ and $P$, respectively. To the intersection of the sets $C_{1}^{u_{1}}, C_{2}^{u_{2}}, \cdots, C_{n}^{u_{n}}$, where the $u_{j}$ are symbols to denote an " $e$," an " $i$," or the complement of an " $e$ " or " $i$," will be assigned the product of the corresponding projections. As the projections are commutative, the order of the terms in the product does not play any role. To a sum $C_{1}^{u_{1}}+C_{2}^{u_{2}}+\cdots+C_{n}^{u_{n}}$ will be assigned the "starred sum" of the corresponding projections. The starred sum of the commutative projections $P_{1}, \cdots, P_{n}$ is defined by $P_{1} \dot{+} \cdots \dot{+} P_{n}=I-\left(I-P_{1}\right) \cdots\left(I-P_{n}\right)$.

(7) Taylor, loc. cit. 
Proceeding in this fashion, it is seen that if a spectral set $\boldsymbol{M}$ is defined in a specific way by the formation of sums and intersections of the elemental sets $C_{j}^{u_{j}}$, a method is specified for forming the projection associated with $\boldsymbol{M}$. That procedure consists in replacing in the definition of $M$ the sets $C_{j}^{\boldsymbol{u}_{j}}$ by their projections, the operation of set intersection by that of projection multiplication, and the operation of set addition by that of projection starred addition. Finally if the projection $P$ is associated with the set $M$, the projection $I-P$ will be associated with the set complementary to $M$. Thus corresponding to a specified construction for a spectral set $M$, there is precisely one projection $P$ defined.

Although the projection associated with $M$ seems to depend on the particular manner in which $M$ is constructed, this is not the case. The proof, which will not be set down, proceeds along these lines. In any two methods of defining $M$, one replaces the auxiliary curves $C_{i}$ by simple polygons $D_{i}$ which are obtained from the $C_{i}$ by deformation. These $D_{i}$ generate in the plane a finite number of polygonal regions $Q_{j}$. The set $\boldsymbol{M}$ by either method of definition consists (up to deformations) of certain of these regions. Finally, any region which lies in one deformation of $\boldsymbol{M}$ but not in the other lies in $R$. Henceforth the projection associated with $\boldsymbol{M}$ will be denoted by $P_{\boldsymbol{M}}$.

THEOREM 9. The mapping $M \rightarrow P_{M}$ of the set algebra of spectral sets on the associated class of projections is a homomorphism. Under the homomorphism $P_{M}=0$ if and only if $M$ lies entirely in the resolvent set of $T$.

The establishment of the homomorphism is virtually accomplished by the discussion just preceding. Let $\boldsymbol{M}_{1}$ and $\boldsymbol{M}_{2}$ be two spectral sets. Then one method of defining the projection associated to $\boldsymbol{M}_{1}+\boldsymbol{M}_{2}$ (or $\boldsymbol{M}_{1} \cdot \boldsymbol{M}_{2}$ ) is $P_{M_{1}+M_{2}}=P_{M_{1}}+P_{M_{2}}\left(P_{M_{1} \cdot M_{2}}=P_{M_{1}} \cdot P_{M_{2}}\right)$. But this is precisely the homomorphism property. It is to be noted that since the projection associated with the whole plane is the identity, the projection $P_{M}$ associated with the set complement $\bar{M}$ of $M$ satisfies $P_{\bar{M}}=I-P_{M}$. That the sets $M$ for which $P_{M}=0$ are precisely those which contain no points of $S$ is a consequence of the uniqueness argument just preceding this theorem.

Spectral components. To obtain an idea of the resolving power of spectral sets, that is to say, of their ability to separate various parts of the spectrum, a few facts concerning sets may be recalled. Since the spectrum of a bounded transformation is closed and compact (or bounded) and since furthermore any closed compact set may serve as the spectrum of some bounded transformation, it is the general closed compact set which must be discussed. If $M$ is such a set and $a$ is any point in $M$, the set sum of all continua in $M$ containing $a$ is a continuum called a component of $M\left({ }^{8}\right)$. Thus $M$ may be expressed as the sum of a finite or infinite number of distinct components. Since a component is a closed compact set, each two components lie at a positive distance

(8) Hausdorff, Mengenlehre, p. 152. 
from each other. Furthermore, if a simple closed curve has no point in common with a given component, that component lies entirely inside or outside the given curve.

If $K_{1}$ and $K_{2}$ are two distinct components (assuming that $M$ consists of more than one component), then there exist closed sets $M_{1}$ and $M_{2}$ such that $M_{1} \cdot M_{2}=0, M=M_{1}+M_{2}, M_{1} \supset K_{1}, M_{2} \supset K_{2}\left({ }^{9}\right)$. Finally, for the sets $M, M_{1}$, and $M_{2}$ there exists a finite set of polygonal domains having the properties: No point of $M$ is on the boundary of any domain; within any domain there is at least one point of $M_{1}$; all the points of $M_{2}$ lie without these domains $\left({ }^{10}\right)$. If $L$ represents a domain containing one point of $K_{1}, L$ contains $K_{1}$ in its entirety. Thus $L$ is a spectral set separating $K_{1}$ from $K_{2}$.

The non-extensibility of the homomorphism. A natural question to propose is whether the class of spectral sets $M$ and the projections $P_{M}$ can be enlarged in such a way that an extended homomorphism reigns between the two extended classes. Specifically, can this extension be carried out in such a fashion that the new homomorphism is valid for denumerable sums and intersections? Preliminary considerations show that it will be wise to forego the insistence that a set $\boldsymbol{M}$ correspond to a projection $P_{M}$ and substitute the requirement that $M$ correspond to a pair of closed linear manifolds $\left\{\mathfrak{M}_{M}, \mathfrak{N}_{M}\right\}$ having the properties: $\mathfrak{M}_{M}$ and $\mathfrak{N}_{M}$ have no common element except 0 ; together they span $\mathfrak{B}$. The notion of a projection $P_{M}$ is a special case of this type where $\mathfrak{M}_{M}$ and $\mathfrak{N}_{M}$ are disjoint $\left.{ }^{11}\right) . \mathfrak{M}_{M}$ is the set of elements $\left\{P_{M} f\right\}$, $f \in \mathfrak{B} ; \mathfrak{N}_{M}$ is the set of elements $\left\{f-P_{M}\right\}, f \in \mathfrak{B}$. The general type of manifold pair described above seems to play the leading role in the theory of rotations-inter alia-in reflexive spaces $\left({ }^{12}\right)$.

Further considerations suggest that the heretofore unqualified nature of the underlying space be restricted suitably so that it should have the "correct" properties relative to infinite intersections and sums of closed linear manifolds. Reflexive spaces seem to possess precisely the requisite properties $\left({ }^{13}\right)$.

(9) R. L. Moore, Foundations of Point Set Theory, American Mathematical Society Colloquium Publications, vol. 13, p. 21, Theorem 35.

(10) Kerékjárto, Vorlesungen ïber Topologie, p. 31.

(11) $\mathfrak{M}_{M}$ and $\mathfrak{R}_{M}$ are disjoint if the elements $f+g, f \in \mathfrak{M}_{M}, g \in \mathfrak{N}_{\boldsymbol{M}}$, are not only dense in $\mathfrak{B}$ but actually fill $\mathfrak{B}$.

(12) See the author's The integral representation of weakly almost-periodic transformations in reflexive vector spaces, these Transactions, vol. 49 (1941), pp. 18-40.

$\left.{ }^{13}\right)$ If $(\mathfrak{B})$ represents the space adjoint to $\mathfrak{B}$, then the reflexive property is defined by $((\mathscr{B}))=\mathfrak{B}$. Thus, for example, the spaces $L_{p}$ and $l_{p}, p>1$, are reflexive. An important property alluded to is the following: If $\left\{\mathfrak{M}_{n}\right\}$ is a monotone decreasing sequence of closed linear manifolds and $\mathfrak{M}_{n}^{\perp}$ represents the largest manifold in $(\mathfrak{B})$ which is orthogonal to $\mathfrak{M}_{n}$, then $\left(\prod_{n=1}^{\infty} \mathfrak{M}_{n}\right)^{\perp}$ $=\sum_{n=1}^{\infty} \mathfrak{M}_{n}^{\perp}$ where $\sum_{n=1}^{\infty} \mathfrak{M}_{n}^{\perp}$ indicates the smallest closed linear manifold containing each $\mathfrak{M}_{n}^{\perp}$.

Proof. Since $\mathfrak{M}_{n}^{\perp} \perp \mathfrak{M}_{n}$, then $\mathfrak{M}_{n}^{\perp} \perp \mathfrak{M}=\prod_{n=1}^{\infty} \mathfrak{M}_{n}$ or $\mathfrak{M}^{\perp} \supset(\mathfrak{R})=\sum_{n=1}^{\infty} \mathfrak{M}_{n}^{\perp}$. Suppose $F \in \mathfrak{M}^{\perp}$, $F \in(\mathfrak{N})$. Then since $\mathfrak{B}$ is reflexive, there exists an $f \in \mathfrak{B}$ such that $F f=1$ and $f \perp(\mathfrak{N})$. In particular, $f \perp \mathfrak{M}_{n}^{\perp}$, hence $f \in \mathfrak{M}_{n}, n=1,2, \cdots$. Thus $f \in \mathfrak{M}$ and $F f=0$. This contradiction proves that $\mathfrak{M}^{\perp}=(\mathfrak{N})$. 
An example will be given of a simple transformation in Hilbert space $\mathfrak{S}$ (which is reflexive) to indicate that the desired extension of the homomorphism is impossible. Let $A$ be the linear transformation defined by the matrix $\left\|a_{i j}\right\|, i, j=0,1,2, \cdots$, with $a_{0 j}=0, j=0,1,2, \cdots ; a_{i 0}=i \cdot 2^{-i}, i=1,2, \cdots$; and $a_{i j}=\delta_{i j} \cdot 2^{-i}, i, j=1,2, \cdots$. Since $\sum_{i, j=0}^{\infty}\left|a_{i j}\right|^{2}<\infty$, the transformation is of finite norm, hence certainly bounded. The spectrum of $A$ is found by examination of the equation

$$
A\left(x_{0}, x_{1}, \cdots\right)-\lambda\left(x_{0}, x_{1}, \cdots\right)=\left(y_{0}, y_{1}, \cdots\right)
$$

where $\sum_{n=0}^{\infty}\left|x_{n}\right|^{2}<\infty, \sum_{n=0}^{\infty}\left|y_{n}\right|^{2}<\infty$. It is found that if $\lambda$ does not assume values in the set $M=\left\{0,1 / 2,1 / 4, \cdots, 1 / 2^{n}, \cdots\right\}$, then (10) has a unique solution $\left(x_{0}, x_{1}, \cdots\right)$ for all $\left(y_{0}, y_{1}, \cdots\right)$. Thus the values in the set complementary to $M$ belong to the resolvent set of $A$. Also, $\lambda=1 / 2^{n}, n=1,2, \cdots$ is found to be a characteristic value for a single vector $\left(x_{0}, x_{1}, \cdots\right)$ where $x_{n}=1$, otherwise $x_{j}=0$. Finally, the value $\lambda=0$ is in the residual spectrum since $A f=0$ implies $f=0$ and since the range of the transformation $A$ is not dense in space.

For the adjoint transformation $\bar{A}$, it is found that the value $\lambda=1 / 2^{n}$, $n=1,2, \cdots$, is in the point spectrum and that the one characteristic vector for that value is the vector $\left(x_{0}, x_{1}, \cdots\right)$ where $x_{0}=1, x_{n}=1 / n$, otherwise $x_{j}=0$. The value $\lambda=0$ is in the point spectrum of $\bar{A}$ and $\bar{A}(1,0,0, \cdots)$ $=(0,0, \cdots)$.

If $C_{n}$ represents a curve containing in its interior all points of $M$ save $1 / 2,1 / 4, \cdots, 1 / 2^{n}$, and if $\bar{P}_{n}$ represents the projection associated with $C_{n}$ and $\bar{A}$, then the manifold $\left\{\bar{P}_{n} f\right\}, f \in \mathfrak{S}$, includes the manifold $\overline{\mathfrak{M}}_{n}$ of all elements of the form $\left(x_{0}, 0,0, \cdots, 0, x_{n+1}, x_{n+2}, \cdots\right)$ by Theorem 8(a). Similarly, the manifold $\left\{f-\bar{P}_{n} f\right\}, f \in \mathfrak{S}$, includes the manifold $\overline{\mathfrak{N}}_{n}$ spanned by the $n$ elements $(1,1 / 2,0,0, \cdots),(1,0,1 / 3,0, \cdots)$ and $(1,0, \cdots, 0,1 / n, 0, \cdots)$. As $\overline{\mathfrak{M}}_{n}$ and $\overline{\mathfrak{R}}_{n}$ span $\mathfrak{S}$, the manifold $\left\{\bar{P}_{n} f\right\}, f \in \mathfrak{S}$ is precisely $\overline{\mathfrak{M}}_{n}$, and the manifold $\left\{f-\bar{P}_{n} f\right\}, f \in \mathfrak{S}$, is precisely $\overline{\mathfrak{N}}_{n}$.

If one writes $\mathfrak{M}_{n}=\overline{\mathfrak{N}}_{n}^{\perp}$ and $\mathfrak{N}_{n}=\overline{\mathfrak{M}}_{n}^{\perp}$, one sees that the projection $P_{n}$ associated with the curve $C_{n}$ and with $A$ determines the manifolds $\mathfrak{M}_{n}$ and $\mathfrak{N}_{n}$. Now $\prod_{n=1}^{\infty} \mathfrak{M}_{n}=\left(\sum_{n=1}^{\infty} \overline{\mathfrak{N}}_{n}\right)^{\perp}=\mathfrak{S}^{\perp}=0$. Also $\sum_{n=1}^{\infty} \mathfrak{N}_{n}=\left(\prod_{n=1}^{\infty} \overline{\mathfrak{M}}_{n}\right)^{\perp}$ $=\left\{\left(x_{0}, 0,0, \cdots\right)\right\}^{\perp} \neq \mathfrak{S}$. This example shows that a homomorphism for denumerable intersections and sums cannot be expected. Also to be noted is that if one attempts to obtain the linear manifold which corresponds to the component of $M$ consisting of the point $\lambda=0$, one obtains the zero manifold, although the transformation has no singularities in this manifold!

As is fairly apparent, the contour integral used as above does not yield all possible projections which reduce $T$. This may be inferred easily from examples. In the first place, there is the possibility of multiplicity in the spectrum. Or even if the spectrum is simple, the integral may not "split" any 
spectral component. Here one may refer for an example to the general rotation in a reflexive space.

There is a way, nevertheless, of obtaining readily a somewhat finer resolution into reducing manifolds than that exhibited above. If $f$ is any element in $\mathfrak{B}$, let $\mathfrak{M}_{f}$ represent the closed linear manifold spanned by the elements $U f$ where $U$ is any function of $T$ (one may let $U$ represent any rational function of $T$ ). Then $T \mathfrak{M}_{f} \subset \mathfrak{M}_{f}$ and one may proceed as above replacing $\mathfrak{B}$ in the previous argument by $\mathfrak{M}_{f}$. It is to be noted that this procedure does not supply une with a manifold complementary to $\mathfrak{M}_{f}$. An obvious approach to problems of the type here envisaged is through a detailed study of the structure of the class of manifolds $\mathfrak{M}_{f}$ where $f$ ranges over $\mathfrak{B}$. This has not yet proved successful.

Barnard College, Columbia University, NEW YORK, N. Y. 